Math. Model. Nat. Phenom.

Vol. 8, No. 5, 2013, pp. 84-94

DOI: $10.1051 / \mathrm{mmnp} / 20138506$

\title{
Moving and Breathing Localized Structures in Reaction-diffusion Systems
}

\author{
S. V. Gurevich ${ }^{*}$, R. Friedrich ${ }^{* *}$ \\ ${ }^{1}$ Institut for Theoretical Physics, University of Münster \\ Wilhelm-Klemm-Str. 9, 48149, Münster, Germany
}

\begin{abstract}
We are interested in the stability of the localized stationary solutions of a threecomponent reaction-diffusion system with one activator and two inhibitors. We show that depending on control parameters, solutions in form of moving and breathing localized structures can be observed in the vicinity of the codimension-two bifurcation point. We analyze this situation performing multiple scale perturbation expansion in the vicinity of the bifurcation point and derive a set of order parameter equations, explicitly describing the dynamics of the single localized structure. Numerical simulations are carried out, showing good agreement with the analytical predictions.
\end{abstract}

Keywords and phrases: complex systems, dissipative solitons, bifurcation analysis

Mathematics Subject Classification: 35B36, 35C08, 70K50

\section{Introduction}

Formation and dynamics of self-organized localized solitary structures, single isolated, randomly distributed or organized in clusters turned out to be of particular interest for fundamental research as well as for applications $[5,12,13]$. In the case of dissipative system we refer to these objects as to dissipative solitons (see $[5,12,13,15]$ and references therein). However, these structures are also variously known as, e.g., oscillons in fluids and granular materials [17,18], ferrosolitons [16] as well as spots and pulses in neuroscience and chemical systems [4,14]. The modeling of dissipative solitons is often carried out via reaction-diffusion systems $[5,14]$. In this paper we focus on the dynamics of dissipative solitons in a three-component reaction-diffusion system with one activator and two inhibitors:

$$
\begin{aligned}
\partial_{t} u & =D_{u} \Delta u+f(u)-\kappa_{3} v-\kappa_{4} w+\kappa_{1}, \\
\tau \partial_{t} v & =D_{v} \Delta v+u-v \\
\theta \partial_{t} w & =D_{w} \Delta w+u-w .
\end{aligned}
$$

Here $u=u(\mathbf{r}, t)$ is the activating component, whereas $v=v(\mathbf{r}, t)$ and $w=w(\mathbf{r}, t)$ denote the inhibiting components and $\mathbf{r}=(x, y)$. In the polynomial function $f(u)=\lambda u-u^{3}$ the coefficient $\lambda$ is positive. $D_{u}, D_{v}, D_{w}$ denote the (positive) diffusion coefficients of the components, whereas the positive parameters

* Corresponding author. E-mail: gurevics@uni-muenster.de

** Deceased 
$\tau$ and $\theta$ represent dimensionless constants, being the ratios of the characteristic times of both inhibitors with respect to that of the activator. In what follows, we use the time constants $\tau$ and $\theta$ as control parameters. The coefficient $\kappa_{1}$ violates the inversion symmetry $(u \mapsto-u)$ and has arbitrary sign. Finally, the constants $\kappa_{3}$ and $\kappa_{4}$ staying in reaction term are also positive, indicating inhibiting nature of $v$ and $w$. The system (1.1) was first introduced in $[1,2]$ as a phenomenological model for a planar dc gas-discharge system with semiconductor electrode. However, (1.1) can be considered as a three-component extension of the FitzHugh-Nagumo system for nerve pulse transmission, which is widely used in various areas of research [3-6]. We are interested in stability properties of a nontrivial stationary solution $\mathbf{q}_{\mathbf{0}}(\mathbf{r})=$ $\left(u_{s}(\mathbf{r}), v_{s}(\mathbf{r}), w_{s}(\mathbf{r})\right)^{T}$ of the system (1.1). In the simplest case it is a stationary localized structure with rotational symmetry (see Fig. 1), which is stable in a certain parameter region. Due to a change in the

(a)

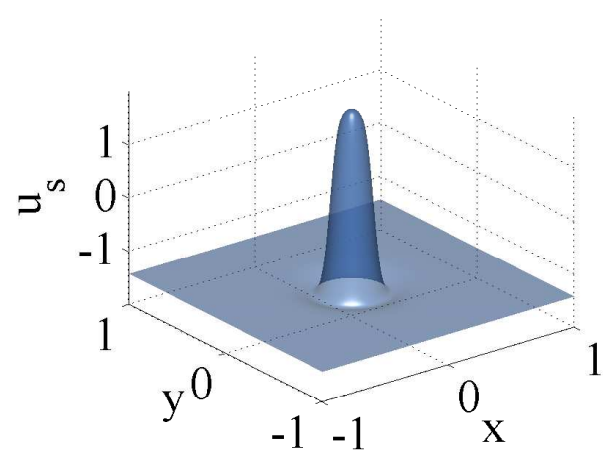

(b)

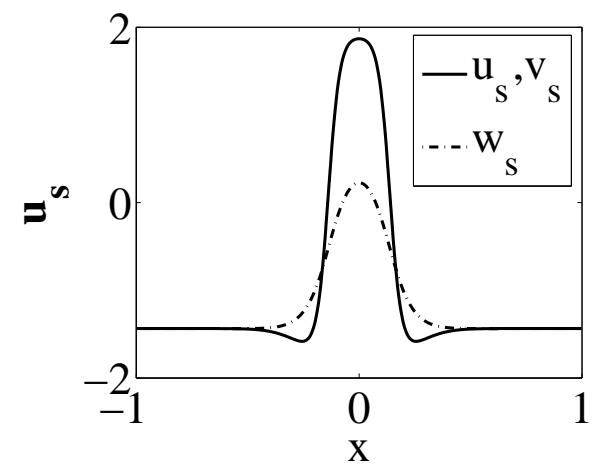

FiguRE 1. Numerical solution of Eq. (1.1) in $\Omega=[-1,1] \times[-1,1]$ with periodic boundary conditions, showing (a) stationary activator distribution $u_{s}(x, y)$ and (b) corresponding cross-sections $u_{s}(x, 0), v_{s}(x, 0), w_{s}(x, 0)$. Parameters: $D_{u}=4.7 \cdot 10^{-3}, D_{v}=0, D_{w}=$ $0.01, \lambda=5.67, \kappa_{1}=-1.04, \kappa_{3}=1, \kappa_{4}=3.33$.

control parameters, a single soliton can lose its stability via, e.g., a drift-bifurcation [8]. Another example is a Hopf bifurcation, leading to a formation of breathing dissipative solitons [7]. Here we are interested in a nontrivial interaction of these instabilities in the vicinity of a codimension-two bifurcation point. We show that this interaction results in a complex dynamical behavior of the soliton, e.g., moving with a constant velocity and breathing with a constant amplitude solution can be observed. We perform a multiple scale perturbation expansion in the vicinity of the bifurcation point and derive a set of order parameter equations, explicitly describing the dynamics of the single dissipative soliton.

\section{Linear stability analysis}

We start from the a reaction-diffusion system in a general form

$$
\frac{\partial}{\partial t} \mathbf{q}(\mathbf{r}, t)=\mathfrak{L}(\nabla, \boldsymbol{\sigma}) \mathbf{q}(\mathbf{r}, t) .
$$

Here $\mathbf{q}=\mathbf{q}(\mathbf{r}, t)=(u, v, w)^{T}$ is a vector-function and $\mathfrak{L}$ is a real-valued nonlinear operator, depending on control parameters $\sigma$.

Let us consider a nontrivial stable stationary solution $\mathbf{q}_{\mathbf{0}}(\mathbf{r})$ of Eq. (2.1). Now if we consider a small perturbation $\mathbf{w}(\mathbf{r}, t)$, so that $\mathbf{q}(\mathbf{r}, t)=\mathbf{q}_{\mathbf{0}}(\mathbf{r})+\mathbf{w}(\mathbf{r}, t)$, the corresponding equation takes the form

$$
\partial_{t} \mathbf{w}=\mathfrak{L}^{\prime}\left(\mathbf{q}_{\mathbf{0}}, \nabla, \boldsymbol{\sigma}\right) \mathbf{w}+\frac{1}{2 !} \mathfrak{L}^{\prime \prime}\left(\mathbf{q}_{\mathbf{0}}, \nabla, \boldsymbol{\sigma}\right) \mathbf{w}: \mathbf{w}+\frac{1}{3 !} \mathfrak{L}^{\prime \prime \prime}\left(\mathbf{q}_{\mathbf{0}}, \nabla, \boldsymbol{\sigma}\right) \mathbf{w}: \mathbf{w}: \mathbf{w}+\ldots,
$$


where we assume that Taylor expansion of $\mathfrak{L}\left(\mathbf{q}_{\mathbf{0}}+\mathbf{w}, \nabla, \boldsymbol{\sigma}\right)$ is possible. Here $\mathfrak{L}^{(n)}\left(\mathbf{q}_{\mathbf{0}}, \nabla, \boldsymbol{\sigma}\right)$ denotes $n^{\prime}$ th Fréchet derivative with respect to $\mathbf{q}$ calculated at $\mathbf{q}=\mathbf{q}_{\mathbf{0}}$. The first coefficient of the expansion (2.2) is a linearization of $\mathfrak{L}$ around the stationary solution $\mathbf{q}_{\mathbf{0}}$, the second term corresponds to the bilinear operator, acting on two perturbation vectors $\mathbf{w}$, etc.

In the following we denote the set of eigenvalues of the linear operator $\mathfrak{L}^{\prime}\left(\mathbf{q}_{\mathbf{0}}, \nabla, \boldsymbol{\sigma}\right)$ as $\mu$ and corresponding eigenfunctions as $\mathcal{F}$, i.e,

$$
\mathfrak{L}^{\prime}\left(\mathbf{q}_{\mathbf{0}}, \nabla, \boldsymbol{\sigma}\right) \mathcal{F}=\mu \mathcal{F}
$$

Notice that generally the operator $\mathfrak{L}^{\prime}\left(\mathbf{q}_{\mathbf{0}}, \nabla, \boldsymbol{\sigma}\right)$ is not self-adjoint, so its eigenvalues and eigenfunctions are typically complex.

If the system (2.1) features translational invariance with respect to spatial coordinates, $\mu=0$ is an eigenvalue of the operator $\mathfrak{L}^{\prime}$, corresponding to two independent neutral eigenfunctions (one for each spatial direction), which in the following will be called Goldstone modes $\mathcal{F}_{\mathbf{r}}^{\mathcal{G}}, \mathbf{r}=(x, y)$. Notice that in systems possessing translational invariance each solution remains a solution if it is shifted to a different spatial position. That is, a small perturbation in the form of Goldstone mode is equivalent to the shift of the stationary solution in the respective direction,

$$
\mathbf{q}_{0}(\mathbf{r}+\epsilon)=\mathbf{q}_{0}(\mathbf{r})+\epsilon \frac{\partial \mathbf{q}_{0}}{\partial \mathbf{r}}+\mathcal{O}\left(\epsilon^{2}\right)
$$

As an infinitesimal shift corresponds to an addition of the spatial derivative of the solution in the respective direction, the Goldstone modes can be identified as the first derivatives of $\mathbf{q}_{\mathbf{0}}$ with respect to the spatial coordinates $\mathbf{r}$, i.e., $\mathcal{F}_{\mathbf{r}}^{\mathcal{G}}=\partial \mathbf{q}_{\mathbf{0}} / \partial \mathbf{r}$.

Stability of $\mathbf{q}_{\mathbf{0}}$ implies that all eigenvalues but $\mu=0$ have negative real parts. The next point to emphasize is that in the case of reaction-diffusion systems the continuous spectrum of the operator $\mathfrak{L}^{\prime}\left(\mathbf{q}_{\mathbf{0}}, \nabla, \boldsymbol{\sigma}\right)$ is separated from zero, i.e., only a finite number of discrete modes, situated near zero can become unstable by the change of control parameters $\sigma$. In order to find these critical modes for a given parameter set one should solve the eigenvalue problem (2.3). For this purpose one can rewrite the system (2.1) in polar coordinates, decompose the small perturbation $\mathbf{w}$ of the radially symmetric stationary solution $\mathbf{q}_{0}$ into a Fourier series and finally rewrite Eq. (2.3) in terms of the amplitude $\mathbf{w}_{\mathbf{n}}$ of the perturbation of the stationary solution with angular dependence $e^{i n \phi}$. Then the influence of the modes with different $n$ on the radial-symmetrical stationary solution $\mathbf{q}_{\mathbf{0}}$ can be understood as follows: the mode with $n=0$ (the breathing mode [7]) results in the change of the size of the soliton; the mode with $n=1$ describes the shift of the solution and $n \geq 2$ causes different deformations of the soliton. An example of real parts of first three critical modes belonging to the discrete spectrum of $\mathfrak{L}^{\prime}\left(\mathbf{q}_{\mathbf{0}}, \nabla, \boldsymbol{\sigma}\right)$ is shown in Fig. 2 (for the mode $n=1$ the corresponding eigenfunction is real).

(a)

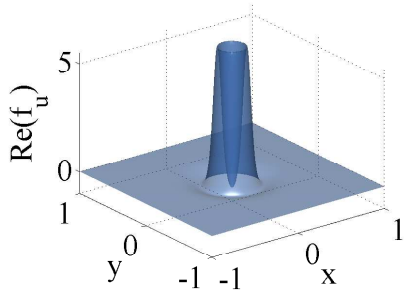

(b)

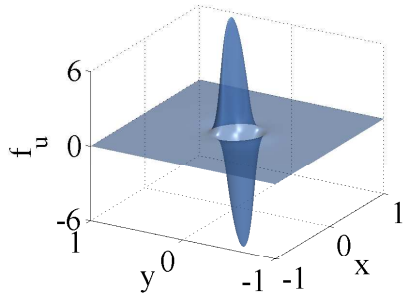

(c)

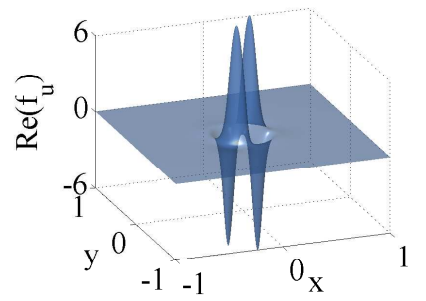

FiguRE 2. Real parts of first three eigenfunctions of Eq. (2.3), corresponding to different $n$. The real parts of the first component of $\mathcal{F}=\left(f_{u}, f_{v}, f_{w}\right)$ are shown. (a) $n=0$, (b) $n=1$, (c) $n=2$. Parameters are the same as in Fig. 1. 


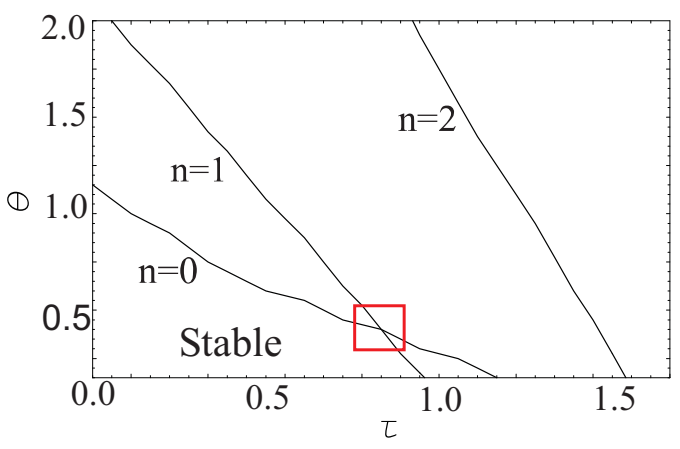

FIgURE 3. Stability diagram in $(\tau, \theta)$ plane, calculated for a solitary solution of the system (1.1). Lines separate stability regions, corresponding to different modes $n=$ $0,1,2$. Parameters are the same as in Fig. 1 . The red square denotes the vicinity of the codimension-two bifurcation point $\left(\tau_{c}, \theta_{c}\right)$.

As mentioned before, the stationary solution qo can lose its stability with the change of one or more control parameters $\sigma$. In what follows, we use the time constants $\tau$ and $\theta$ as control parameters, i.e, $\boldsymbol{\sigma}=\left(\sigma_{1}, \sigma_{2}\right)$, where $\sigma_{1}=\tau$ and $\sigma_{2}=\theta$. This is convenient because the stationary solution does not depend on them. Now we can solve the eigenvalue problem (2.3) numerically for different values of the control parameters $\tau$ and $\theta$, keeping all other parameters fixed. The resulting stability diagram in $(\tau, \theta)$ plane can be seen in Fig. 3. One can easily see that for small values of $\tau$ and $\theta$ the stationary solution is stable. An increase of the constants leads to the excitation of either $n=0$ or $n=1$ mode. The modes $n \geq 2$ become unstable for larger value of $\tau$ and $\theta$ and cannot be responsible for the primary destabilizaion of $\mathbf{q}_{\mathbf{0}}$. That is, two primary destabilization scenarios can be observed. According to the first one a pair of complex-conjugated eigenvalues passes through the imaginary axis as the control parameter $\theta$ exceeds some critical value $\theta_{c}$. In this case the stationary solution qo bifurcates to an oscillatory breathing state via the Hopf bifurcation. The resulting pattern can oscillate with the constant amplitude or the oscillation amplitude increases, what leads to a collapse of the solution [7]. The second destabilization scenario corresponds to the case of the so-called drift bifurcation: The critical eigenvalue passes through zero for some $\tau=\tau_{c}$, so that the corresponding critical eigenfunction can be represented as a linear combination of the Goldstone modes. The system of eigenfunctions is then incomplete and has to be supplemented with a generalized eigenfunction with the eigenvalue $\mu=0$, which we refer to as propagator mode $\mathcal{F}_{\boldsymbol{r}}^{\mathcal{P}}[8,11]$,

$$
\mathfrak{L}_{c}^{\prime} \mathcal{F}_{\boldsymbol{r}}^{\mathcal{P}}=\mu \mathcal{F}_{\boldsymbol{r}}^{\mathcal{P}}+\mathcal{F}_{\boldsymbol{r}}^{\mathcal{G}}
$$

or, equivalently,

$$
\mathfrak{L}_{c}^{\prime} \mathcal{F}_{\boldsymbol{r}}^{\mathcal{P}}=\mathcal{F}_{\boldsymbol{r}}^{\mathcal{G}},
$$

where $\mathfrak{L}_{c}^{\prime}:=\mathfrak{L}^{\prime}\left(\mathbf{q}_{\mathbf{0}}, \nabla, \boldsymbol{\sigma}_{\boldsymbol{c}}\right)$. The stationary solution $\mathbf{q}_{0}$ becomes unstable at the point $\tau=\tau_{c}$ due to existence of the mode $\mathcal{F}_{r}^{\mathcal{P}}$ : a perturbation in the form of the propagator mode causes a change in the form of the Goldstone mode that corresponds to a permanent shift of the solution [8]. Hence one is concerned with a transition from a stationary to a moving state, i.e., a drift bifurcation. Notice also that in accordance with the Fredholm alternative Eq. (2.4) can be solved if and only if the equality

$$
\left\langle\mathcal{F}_{\boldsymbol{r}}^{\mathcal{G} *} \mid \mathcal{F}_{\boldsymbol{r}}^{\mathcal{G}}\right\rangle=0
$$

is fulfilled, where $\mathcal{F}_{\boldsymbol{r}}^{\mathcal{G} *}$ is an eigenfunction of the adjoint operator $\mathfrak{L}_{c}^{\dagger}$ to the eigenvalue zero and the same spatial symmetry as $\mathcal{F}_{\boldsymbol{r}}^{\mathcal{G}}$ and $\langle\cdot \mid \cdot\rangle$ denotes the scalar product defined in terms of full spatial integration over the considered domain, like e.g., in [8-10]. Notice that in general the condition (2.5) is not fulfilled and becomes correct only in degeneracy point $\tau=\tau_{c}$, where instability takes place. 


\section{Direct numerical simulations}

We are interested in the behavior of the system in the vicinity of the codimension-two bifurcation point $\boldsymbol{\sigma}_{\boldsymbol{c}}=\left(\sigma_{1 c}, \sigma_{2 c}\right)=\left(\tau_{c}, \theta_{c}\right)$, where Hopf- and drift-bifurcations take place simultaneously (see Fig. 3$)$. We have performed direct numerical simulations of the system (1.1) in the vicinity of the bifurcation point $\sigma_{c}$. The result of the simulations is depicted in Fig. 4. If $\theta$ is chosen to be close to $\theta_{c}$, an initial pulse,

(a)

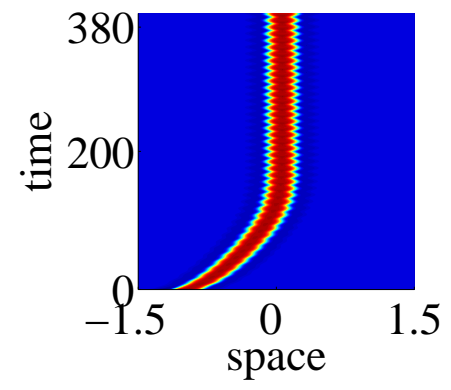

(c)

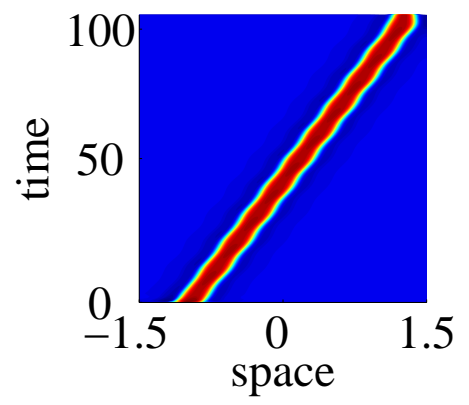

(b)

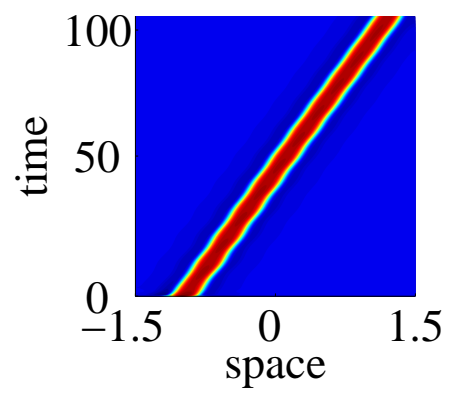

(d)

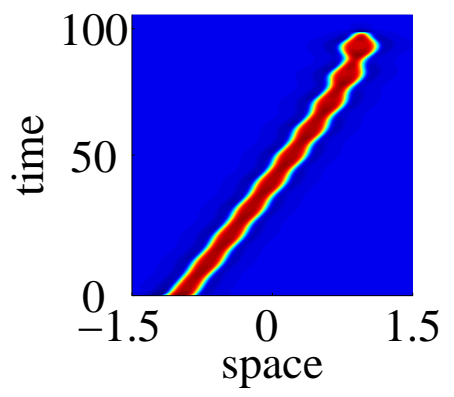

FiguRE 4. Space-time plots of the activator distribution $u$ for different values of $\tau$ and $\theta$ obtained from numerical solution of (1.1). Time evolution of the cross-section of the initial pulse close to the stationary solution is shown. (a) $\tau=0.834, \theta=0.4$; (b) $\tau=0.9$, $\theta=0.43$; (c) $\tau=0.9, \theta=0.46$; (d) $\tau=0.9, \theta=0.48$. Other parameters: $D_{u}=4.7 \cdot 10^{-3}$, $D_{v}=0, D_{w}=0.01, \lambda=5.67, \kappa_{1}=-1.04, \kappa_{3}=1.0, \kappa_{4}=3.33, \tau=0.834$. The bifurcation point is $\left(\tau_{c}, \theta_{c}\right)=(0.834,0.376)$. The calculations were performed on the rectangular domain $\Omega=[-1.5,1.5] \times[-1.5,1.5]$ with periodic boundary conditions.

which shape is close to the stationary solution, converges to moving and breathing dissipative soliton, as shown in Fig. 4 (a). In this case in the initial phase of the evolution the displacement of the soliton is proportional to $\sqrt{t}$, whereas the oscillation amplitude increases. However, after some time the soliton stops, and oscillation amplitude reaches some constant value. So finally one obtains a stationary soliton, breathing with a constant amplitude. Figure 4 (b)-(d) shows other instability scenarios. In contrast to the first scenario, here the soliton moves with a constant velocity. The oscillation amplitude decreases, so finally one has just a dissipative soliton, moving with a constant velocity (Fig. 4 (b)). Another example is shown in Fig. 4 (c). Here initial pulse converges to the soliton, moving with the constant velocity and breathing with a constant amplitude. Further increase of the one of the control parameters destroys the solution (see Fig. 4 (d)).

The simulations indicate the difference in dynamics of single dissipative soliton in the vicinity of the codimension-two bifurcation point. This may be explained with the fact that the corresponding critical eigenmodes $n=0$ and $n=1$ have different increments, and consequently different influences on the 
dynamics of the solution. In the first case (Fig. 4 (a)) the mode $n=0$ seems to be dominant, whereas in the rest of simulations the mode $n=1$ determines essentially the behavior of the system.

In order to investigate the stability of the soliton in the vicinity of the bifurcation point $\left(\sigma_{1 c}, \sigma_{2 c}\right)$ from the point of view of bifurcation theory, we represent the real-valued vector function $\mathbf{q}(\mathbf{r}, t)$ at the bifurcation point as

$$
\begin{aligned}
\mathbf{q}(\mathbf{r}, t)= & \mathbf{q}_{\mathbf{0}}(\mathbf{r}-\mathbf{R})+\boldsymbol{\xi} \mathcal{F}_{r}^{\mathcal{P}}(\mathbf{r}-\mathbf{R})+\boldsymbol{\eta} e^{i \omega t} \mathcal{F}_{\boldsymbol{r}}^{\mathcal{B}}(\mathbf{r}-\mathbf{R})+ \\
& +\mathcal{Y}(\mathbf{r}-\mathbf{R}) e^{2 i \omega t}+\text { c.c. }+\mathbf{s}(\mathbf{r}-\mathbf{R}),
\end{aligned}
$$

where $\mathbf{R}$ represents a constant position of the stationary solution $\mathbf{q}_{\mathbf{0}}, \boldsymbol{\xi}$ is a constant amplitude of the propagator mode $\mathcal{F}_{\boldsymbol{r}}^{\mathcal{P}}$, whereas $\boldsymbol{\eta}$ denotes a constant complex amplitude of the breathing mode $\mathcal{F}_{\boldsymbol{r}}^{\mathcal{B}}$. In addition $\mathcal{Y}$ stays for the contribution of the second harmonics and $\mathbf{s}(\mathbf{r}, t)$ represents a sum of the stable fast decaying modes.

Now if we increase the control parameters, $\sigma_{1}=\sigma_{1 c}+\varepsilon_{1}, \sigma_{2}=\sigma_{2 c}+\varepsilon_{2}, \varepsilon_{1,2} \ll 1$, the amplitudes $\boldsymbol{\xi}$ and $\boldsymbol{\eta}$ as well as the position $\mathbf{R}$ become slow functions of time, i.e.,

$$
\begin{aligned}
\mathbf{q}= & \mathbf{q}_{\mathbf{0}}(\mathbf{r}-\mathbf{R}(t))+\boldsymbol{\xi}(t) \mathcal{F}_{\boldsymbol{r}}^{\mathcal{P}}(\mathbf{r}-\mathbf{R}(t))+\boldsymbol{\eta}(t) e^{i \omega t} \mathcal{F}_{\boldsymbol{r}}^{\mathcal{B}}(\mathbf{r}-\mathbf{R}(t))+ \\
& +\mathcal{Y}(\mathbf{r}-\mathbf{R}(t)) e^{2 i \omega t}+\text { c.c. }+\mathbf{s}(\mathbf{r}-\mathbf{R}(t), t) .
\end{aligned}
$$

Now our goal is to write down a set of ordinary differential equations for the unstable amplitudes $\boldsymbol{\xi}(t)$, $\boldsymbol{\eta}(t)$ and position $\mathbf{R}(t)$ that describe the behavior of the single soliton solution in the vicinity of the bifurcation point $\left(\sigma_{1 c}, \sigma_{2 c}\right)$.

\section{Order parameter equations}

\subsection{Order parameter equations in general form}

In the following, without loss of generality we will restrict ourselves to the case $\mathbf{r}=x$, as all spatial directions are equal, i.e., the perturbed solution will move only in the $x$-direction. Moreover, for the sake of simplicity we will drop the subindices $\mathbf{r}$ of all critical eigenfunctions. Substituting the ansatz (3.2) into Eq. (2.2) and equalizing the terms with the frequencies $\omega$ and zero, respectively, we obtain:

$$
\begin{aligned}
& \dot{\boldsymbol{\eta}} \mathcal{F}^{\mathcal{B}}-\dot{\mathbf{R}} \boldsymbol{\eta} \frac{\partial \mathcal{F}^{\mathcal{B}}}{\partial r}=\boldsymbol{\eta}\left(\varepsilon_{1} \mathcal{L}_{1}^{\prime} \mathcal{F}^{\mathcal{B}}+\varepsilon_{2} \mathcal{L}_{2}^{\prime} \mathcal{F}^{\mathcal{B}}\right)+\boldsymbol{\xi} \boldsymbol{\eta} \mathcal{L}_{c}^{\prime \prime} \mathcal{Y} \overline{\mathcal{F}}^{\mathcal{B}}+\overline{\boldsymbol{\eta}} \mathcal{L}_{c}^{\prime \prime} \mathcal{Y} \overline{\mathcal{F}}^{\mathcal{B}}+ \\
& \boldsymbol{\eta} \mathcal{L}_{c}^{\prime \prime} \mathcal{F}^{\mathcal{B}} \mathbf{s}+\frac{\boldsymbol{\xi}^{2} \boldsymbol{\eta}}{2} \mathcal{L}_{c}^{\prime \prime \prime} \mathcal{F}^{\mathcal{P}} \mathcal{F}^{\mathcal{P}} \mathcal{F}^{\mathcal{B}}+\frac{|\boldsymbol{\eta}|^{2} \boldsymbol{\eta}}{2} \mathcal{L}_{c}^{\prime \prime \prime} \mathcal{F}^{\mathcal{B}} \mathcal{F}^{\mathcal{B}} \overline{\mathcal{F}}^{\mathcal{B}} ; \\
& -\dot{\mathbf{R}} \mathcal{F}^{\mathcal{G}}+\dot{\boldsymbol{\xi}} \mathcal{F}^{\mathcal{P}}-\dot{\mathbf{R}} \dot{\boldsymbol{\xi}} \frac{\partial \mathcal{F}^{\mathcal{P}}}{\partial \mathbf{r}}+\frac{\partial \mathbf{s}}{\partial t}-\dot{\mathbf{R}} \frac{\partial \mathbf{s}}{\partial \boldsymbol{r}}=\boldsymbol{\xi} \mathcal{F}^{\mathcal{G}}+\mathcal{L}_{c}^{\prime} \mathbf{s}+ \\
& \boldsymbol{\xi}\left(\varepsilon_{1} \mathcal{L}_{1}^{\prime} \mathcal{F}^{\mathcal{P}}+\varepsilon_{2} \mathcal{L}_{2}^{\prime} \mathcal{F}^{\mathcal{P}}\right)+\frac{\boldsymbol{\xi}^{2}}{2} \mathcal{L}_{c}^{\prime \prime} \mathcal{F}^{\mathcal{P}} \mathcal{F}^{\mathcal{P}}+|\boldsymbol{\eta}|^{2} \mathcal{L}_{c}^{\prime \prime} \mathcal{F}^{\mathcal{B}} \overline{\mathcal{F}}^{\mathcal{B}}+\boldsymbol{\xi} \mathcal{L}_{c}^{\prime \prime} \mathcal{F}^{\mathcal{P}} \mathbf{s}+ \\
& \frac{\boldsymbol{\xi}^{3}}{6} \mathcal{L}_{c}^{\prime \prime \prime} \mathcal{F}^{\mathcal{P}} \mathcal{F}^{\mathcal{P}} \mathcal{F}^{\mathcal{P}}+\boldsymbol{\xi}|\boldsymbol{\eta}|^{2} \mathcal{L}_{c}^{\prime \prime \prime} \mathcal{F}^{\mathcal{P}} \mathcal{F}^{\mathcal{B}} \overline{\mathcal{F}}^{\mathcal{B}} .
\end{aligned}
$$

Here $\mathcal{L}_{1}^{\prime}=\left.\frac{\partial \mathcal{L}^{\prime}\left(\mathbf{q}_{0}, \nabla, \sigma\right)}{\partial \sigma_{1}}\right|_{\sigma_{1}=\sigma_{1 c}}$ and $\mathcal{L}_{2}^{\prime}=\left.\frac{\partial \mathcal{L}^{\prime}\left(\mathbf{q}_{0}, \nabla, \sigma\right)}{\partial \sigma_{2}}\right|_{\sigma_{2}=\sigma_{2 c}}$ represent deviations of the linearization operator from the $\mathcal{L}_{c}^{\prime}:=\mathcal{L}^{\prime}\left(\mathbf{q}_{0}, \nabla, \sigma_{1 c}, \sigma_{2 c}\right)$, so that

$$
\mathcal{L}^{\prime}\left(\mathbf{q}_{0}, \nabla, \sigma_{1 c}+\varepsilon_{1}, \sigma_{2 c}+\varepsilon_{2}\right)=\mathcal{L}_{c}^{\prime}+\varepsilon_{1} \mathcal{L}_{1}^{\prime}+\varepsilon_{2} \mathcal{L}_{2}^{\prime} .
$$

In addition, equalizing the terms with the frequency $2 i \omega$, we obtain the equation

$$
\frac{\partial \mathcal{Y}}{\partial t}+2 i \omega=\mathcal{L}_{c}^{\prime} \mathcal{Y}+\frac{1}{2} \mathcal{L}_{c}^{\prime \prime} \mathcal{F}^{\mathcal{B}} \mathcal{F}^{\mathcal{B}}
$$


Notice that the function $\mathcal{Y}$ describes the contribution of the second harmonics and belongs to the stable modes. That is, the time derivative of $\mathcal{Y}$ can be eliminated adiabatically [19] and the amplitude $\mathcal{Y}$ can be expressed in terms of unstable amplitude $\boldsymbol{\eta}$ as $\mathcal{Y}=\boldsymbol{\eta}^{2} \mathcal{X}$, where $\mathcal{X}$ denotes a function living in the same space as the eigenfunction $\mathcal{F}^{\mathcal{B}}$ and $\boldsymbol{\eta}^{2}$ is the corresponding amplitude. Then, one obtains the solvability condition with respect to the unknown function $\mathcal{X}$ :

$$
\left(2 i \omega-\mathcal{L}_{c}^{\prime}\right) \mathcal{X}=\frac{1}{2} \mathcal{L}_{c}^{\prime \prime} \mathcal{F}^{\mathcal{B}} \mathcal{F}^{\mathcal{B}}
$$

Now, in order to write the equations for the amplitudes from the achieved relations (4.1), (4.2), the state vectors have to be projected. As the operator $\mathcal{L}_{c}^{\prime}$ is not self-adjoint, its eigenfunctions do not obey an orthogonality relation. Instead, the components of the modes have to be calculated by projection on the corresponding modes of the adjoint operator $\mathcal{L}_{c}^{\prime \dagger}$. First, we project Eq. (4.1) onto the mode $\mathcal{F}^{\mathcal{B} \dagger}$ of the operator $\mathcal{L}_{c}^{\prime \dagger}$, corresponding to the critical eigenvalue $-i \omega$. The projection yields:

$$
\begin{aligned}
& \dot{\boldsymbol{\eta}}=\left(\varepsilon_{1} \frac{\left\langle\mathcal{L}_{1}^{\prime} \mathcal{F}^{\mathcal{B}} \mid \mathcal{F}^{\mathcal{B} \dagger}\right\rangle}{\left\langle\mathcal{F}^{\mathcal{B}} \mid \mathcal{F}^{\mathcal{B} \dagger}\right\rangle}+\varepsilon_{2} \frac{\left\langle\mathcal{L}_{2}^{\prime} \mathcal{F}^{\mathcal{B}} \mid \mathcal{F}^{\mathcal{B} \dagger}\right\rangle}{\left\langle\mathcal{F}^{\mathcal{B}} \mid \mathcal{F}^{\mathcal{B} \dagger}\right\rangle}\right) \boldsymbol{\eta}+\frac{\left\langle\mathcal{L}_{c}^{\prime \prime} \mathcal{F}^{\mathcal{B}} \mid \mathcal{F}^{\mathcal{B} \dagger}\right\rangle}{\left\langle\mathcal{F}^{\mathcal{B}} \mid \mathcal{F}^{\mathcal{B}}\right\rangle} \boldsymbol{\eta}+ \\
& \left(\frac{\left\langle\mathcal{L}_{c}^{\prime \prime \prime} \mathcal{F}^{\mathcal{B}} \mathcal{F}^{\mathcal{B}} \overline{\mathcal{F}}^{\mathcal{B}} \mid \mathcal{F}^{\mathcal{B} \dagger}\right\rangle}{2\left\langle\mathcal{F}^{\mathcal{B}} \mid \mathcal{F}^{\mathcal{B} \dagger}\right\rangle}+\frac{\left\langle\mathcal{L}_{c}^{\prime \prime} \mathcal{X} \overline{\mathcal{F}}^{\mathcal{B}} \mid \mathcal{F}^{\mathcal{B} \dagger}\right\rangle}{\left\langle\mathcal{F}^{\mathcal{B}} \mid \mathcal{F}^{\mathcal{B} \dagger}\right\rangle}\right)|\boldsymbol{\eta}|^{2} \boldsymbol{\eta}+\frac{\left\langle\mathcal{L}_{c}^{\prime \prime \prime} \mathcal{F}^{\mathcal{P}} \mathcal{F}^{\mathcal{P}} \mathcal{F}^{\mathcal{B}} \mid \mathcal{F}^{\mathcal{B} \dagger}\right\rangle}{2\left\langle\mathcal{F}^{\mathcal{B}} \mid \mathcal{F}^{\mathcal{B}^{\dagger}}\right\rangle} \boldsymbol{\xi}^{2} \boldsymbol{\eta} .
\end{aligned}
$$

Note that $\mathcal{F}^{\mathcal{B}}$ is orthogonal to all modes of the operator $\mathcal{L}_{c}^{\prime}$, but $\mathcal{F}^{\mathcal{B}}$. In order to obtain the equations for $\boldsymbol{\xi}(t)$ and $\mathbf{R}(t)$, we project Eq. (4.2) onto the eigenfunction $\mathcal{F}^{\mathcal{G} \dagger}$ of the adjoint operator with the eigenvalue zero as well as onto the generalized eigenfunction $\mathcal{F}^{\mathcal{P} \dagger}$ of the operator $\mathcal{L}_{c}^{\prime \dagger}$, corresponding to the same zero eigenvalue, i.e.,

$$
\mathcal{L}_{c}^{\prime \dagger} \mathcal{F}^{\mathcal{P} \dagger}=\mathcal{F}^{\mathcal{G} \dagger}
$$

The projection onto $\mathcal{F}^{\mathcal{P} \dagger}$ provides the dynamical equation for the position $\mathbf{R}(t)$ :

$$
\begin{aligned}
& \dot{\mathbf{R}}=-\left(1+\varepsilon_{1} \frac{\left\langle\mathcal{F}^{\mathcal{P} \dagger} \mid \mathcal{L}_{1}^{\prime} \mathcal{F}^{\mathcal{P}}\right\rangle}{\left\langle\mathcal{F}^{\mathcal{P} \dagger} \mid \mathcal{F}^{\mathcal{G}}\right\rangle}+\varepsilon_{2} \frac{\left\langle\mathcal{F}^{\mathcal{P} \dagger} \mid \mathcal{L}_{2}^{\prime} \mathcal{F}^{\mathcal{P}}\right\rangle}{\left\langle\mathcal{F}^{\mathcal{P}} \mid \mathcal{F}^{\mathcal{G}}\right\rangle}\right) \boldsymbol{\xi}-\frac{2\left\langle\frac{\partial \mathcal{F}^{\mathcal{P} \dagger}}{\partial \mathbf{r}} \mid \mathbf{s}\right\rangle}{\left\langle\mathcal{F}^{\mathcal{P}} \mid \mathcal{F}^{\mathcal{G}}\right\rangle} \boldsymbol{\xi}- \\
& \frac{\left\langle\mathcal{F}^{\mathcal{P} \dagger} \mid \mathcal{L}_{c}^{\prime \prime} \mathcal{F}^{\mathcal{P}} \mathbf{s}\right\rangle}{\left\langle\mathcal{F}^{\mathcal{P} \dagger} \mid \mathcal{F}^{\mathcal{G}}\right\rangle} \boldsymbol{\xi}-\frac{\left\langle\mathcal{F}^{\mathcal{P}} \mid \mathcal{L}_{c}^{\prime \prime \prime} \mathcal{F}^{\mathcal{P}} \mathcal{F}^{\mathcal{P}} \mathcal{F}^{\mathcal{P}}\right\rangle}{6\left\langle\mathcal{F}^{\mathcal{P}} \mid \mathcal{F}^{\mathcal{G}}\right\rangle} \boldsymbol{\xi}^{3}-\frac{\left\langle\mathcal{F}^{\mathcal{P}} \mid \mathcal{L}_{c}^{\prime \prime \prime} \mathcal{F}^{\mathcal{P}} \mathcal{F}^{\mathcal{B}} \overline{\mathcal{F}}^{\mathcal{B}}\right\rangle}{\left\langle\mathcal{F}^{\mathcal{P} \dagger} \mid \mathcal{F}^{\mathcal{G}}\right\rangle} \boldsymbol{\xi}|\boldsymbol{\eta}|^{2}
\end{aligned}
$$

The last projection Eq. (4.2) onto the eigenfunction $\mathcal{F}^{\mathcal{G} \dagger}$ together with the relation

$$
\left\langle\mathcal{F}^{\mathcal{G} \dagger} \mid \frac{\partial \mathbf{s}}{\partial t}\right\rangle=\dot{\mathbf{R}}\left\langle\frac{\partial \mathcal{F}^{\mathcal{G} \dagger}}{\partial \mathbf{r}} \mid \mathbf{s}\right\rangle
$$

and Eq. (4.5) gives

$$
\begin{aligned}
& \dot{\boldsymbol{\xi}}=\left(\varepsilon_{1} \frac{\left\langle\mathcal{F}^{\mathcal{G}} \mid \mathcal{L}_{1}^{\prime} \mathcal{F}^{\mathcal{P}}\right\rangle}{\left\langle\mathcal{F}^{\mathcal{G} \dagger} \mid \mathcal{F}^{\mathcal{P}}\right\rangle}+\varepsilon_{2} \frac{\left\langle\mathcal{F}^{\mathcal{G}} \mid \mathcal{L}_{2}^{\prime} \mathcal{F}^{\mathcal{P}}\right\rangle}{\left\langle\mathcal{F}^{\mathcal{G}} \mid \mathcal{F}^{\mathcal{P}}\right\rangle}\right) \boldsymbol{\xi}+\frac{\left\langle\mathcal{F}^{\mathcal{G}} \mid \mathcal{L}_{c}^{\prime \prime} \mathcal{F}^{\mathcal{P}} \mathbf{s}\right\rangle}{\left\langle\mathcal{F}^{\dagger} \mid \mathcal{F}^{\mathcal{P}}\right\rangle} \boldsymbol{\xi}+ \\
& \frac{2 a_{1}\left\langle\frac{\partial \mathcal{F}^{\mathcal{G} \dagger}}{\partial \mathbf{r}} \mid \mathbf{s}\right\rangle}{\left\langle\mathcal{F}^{\mathcal{G} \dagger} \mid \mathcal{F}^{\mathcal{P}}\right\rangle} \boldsymbol{\xi}+\frac{\left\langle\mathcal{F}^{\dagger} \dagger \mid \mathcal{L}_{c}^{\prime \prime \prime} \mathcal{F}^{\mathcal{P}} \mathcal{F}^{\mathcal{P}} \mathcal{F}^{\mathcal{P}}\right\rangle}{6\left\langle\mathcal{F}^{\dagger} \dagger \mid \mathcal{F}^{\mathcal{P}}\right\rangle} \boldsymbol{\xi}^{3}+\frac{\left\langle\mathcal{F}^{\dagger} \mid \mathcal{L}_{c}^{\prime \prime \prime} \mathcal{F}^{\mathcal{P}} \mathcal{F}^{\mathcal{P}} \overline{\mathcal{F}}^{\mathcal{B}}\right\rangle}{\left\langle\mathcal{F}^{\dagger} \mid \mathcal{F}^{\mathcal{P}}\right\rangle} \boldsymbol{\xi}|\boldsymbol{\eta}|^{2},
\end{aligned}
$$

where

$$
a_{1}=1+\varepsilon_{1} \frac{\left\langle\mathcal{F}^{\mathcal{P} \dagger} \mid \mathcal{L}_{1}^{\prime} \mathcal{F}^{\mathcal{P}}\right\rangle}{\left\langle\mathcal{F}^{\mathcal{P} \dagger} \mid \mathcal{F}^{\mathcal{G}}\right\rangle}+\varepsilon_{2} \frac{\left\langle\mathcal{F}^{\mathcal{P}} \mid \mathcal{L}_{2}^{\prime} \mathcal{F}^{\mathcal{P}}\right\rangle}{\left\langle\mathcal{F}^{\mathcal{P} \dagger} \mid \mathcal{F}^{\mathcal{G}}\right\rangle}
$$

stands for the linear coefficient of (4.5).

Equations (4.4), (4.5) and (4.6) are desired dynamical equations for $\boldsymbol{\eta}(t), \boldsymbol{\xi}(t)$ and $\mathbf{R}(t)$. However, they contain the unknown function $\mathbf{s}(\mathbf{r}, t)$, which represents the sum of fast relaxing stable modes. Since the amplitudes of stable modes are fast relaxing variables, their long time behavior is defined by the 
behavior of the amplitudes of unstable modes. That is, applying the general procedure of adiabatic elimination [19], the function $\mathbf{s}$ can be found from Eqn. (4.2) and (4.5) as

$$
\mathbf{s}=\mathcal{Z} \boldsymbol{\xi}^{2}+\mathcal{T}|\boldsymbol{\eta}|^{2}
$$

where $\mathcal{Z}$ and $\mathcal{T}$ represent the solutions of the linear inhomogeneous equations

$$
\begin{aligned}
& \mathfrak{L}_{c}^{\prime} \mathcal{Z}=a_{1} \frac{\partial \mathcal{F}_{\boldsymbol{r}}^{\mathcal{P}}}{\partial \mathbf{r}}-\frac{1}{2} \mathfrak{L}_{c}^{\prime \prime} \mathcal{F}_{\boldsymbol{r}}^{\mathcal{P}} \mathcal{F}_{\boldsymbol{r}}^{\mathcal{P}}, \\
& \mathfrak{L}_{c}^{\prime} \mathcal{T}=-\mathfrak{L}_{c}^{\prime \prime} \mathcal{F}_{\boldsymbol{r}}^{\mathcal{B}} \frac{\mathcal{F}_{\boldsymbol{r}}^{\mathcal{B}}}{}
\end{aligned}
$$

Notice that due to symmetry properties the inhomogeneous part of Eqn. (4.8)-(4.9) is orthogonal to the kernel of the operator $\mathfrak{L}_{c}^{\prime \dagger}$. That is, according to the Fredholm alternative the unique solutions of (4.8)-(4.9) always exist.

Altogether, the order parameter equations for $\boldsymbol{\eta}, \boldsymbol{\xi}$ and $\mathbf{R}$ take the form:

$$
\begin{aligned}
\dot{\mathbf{R}} & =a_{1} \boldsymbol{\xi}+a_{2} \boldsymbol{\xi}|\boldsymbol{\eta}|^{2}+a_{3} \boldsymbol{\xi}^{3}, \\
\dot{\boldsymbol{\eta}} & =\left(\varepsilon_{1} b_{1}+\varepsilon_{2} b_{2}\right) \boldsymbol{\eta}+b_{3}|\boldsymbol{\eta}|^{2} \boldsymbol{\eta}+b_{4} \boldsymbol{\xi}^{2} \boldsymbol{\eta}, \\
\dot{\boldsymbol{\xi}} & =\left(\varepsilon_{1} c_{1}+\varepsilon_{2} c_{2}\right) \boldsymbol{\xi}+c_{3} \boldsymbol{\xi}^{3}+c_{4} \boldsymbol{\xi}|\boldsymbol{\eta}|^{2},
\end{aligned}
$$

where all complex coefficients can be found as:

$$
\begin{aligned}
& a_{2}=\frac{2\left\langle\frac{\partial \mathcal{F}^{\mathcal{P} \dagger}}{\partial \mathbf{r}} \mid \mathcal{T}\right\rangle}{\left\langle\mathcal{F}^{\mathcal{P} \dagger} \mid \mathcal{F}^{\mathcal{G}}\right\rangle}+\frac{\left\langle\mathcal{F}^{\mathcal{P}^{\dagger}} \mid \mathcal{L}_{c}^{\prime \prime} \mathcal{F}^{\mathcal{P}} \mathcal{T}\right\rangle}{\left\langle\mathcal{F}^{\mathcal{P} \dagger} \mid \mathcal{F}^{\mathcal{G}}\right\rangle}+\frac{\left\langle\mathcal{F}^{\mathcal{P} \dagger} \mid \mathcal{L}_{c}^{\prime \prime \prime} \mathcal{F}^{\mathcal{P}} \mathcal{F}^{\mathcal{B}} \overline{\mathcal{F}^{\mathcal{B}}}\right\rangle}{\left\langle\mathcal{F}^{\mathcal{P}} \mid \mathcal{F}^{\mathcal{G}}\right\rangle}, \\
& a_{3}=\frac{2\left\langle\frac{\partial \mathcal{F}^{\mathcal{P} \dagger}}{\partial \mathbf{r}} \mid \mathcal{Z}\right\rangle}{\left\langle\mathcal{F}^{\mathcal{P} \dagger} \mid \mathcal{F}^{\mathcal{G}}\right\rangle}+\frac{\left\langle\mathcal{F}^{\mathcal{P} \dagger} \mid \mathcal{L}_{c}^{\prime \prime} \mathcal{F}^{\mathcal{P}} \mathcal{Z}\right\rangle}{\left\langle\mathcal{F}^{\mathcal{P} \dagger} \mid \mathcal{F}^{\mathcal{G}}\right\rangle}+\frac{\left\langle\mathcal{F}^{\mathcal{P} \dagger} \mid \mathcal{L}_{c}^{\prime \prime \prime} \mathcal{F}^{\mathcal{P}} \mathcal{F}^{\mathcal{P}} \mathcal{F}^{\mathcal{P}}\right\rangle}{6\left\langle\mathcal{F}^{\mathcal{P} \dagger} \mid \mathcal{F}^{\mathcal{G}}\right\rangle} ; \\
& b_{1}=\frac{\left\langle\mathcal{L}_{1}^{\prime} \mathcal{F}^{\mathcal{B}} \mid \mathcal{F}^{\mathcal{B} \dagger}\right\rangle}{\left\langle\mathcal{F}^{\mathcal{B}} \mid \mathcal{F}^{\mathcal{B} \dagger}\right\rangle}, \quad b_{2}=\frac{\left\langle\mathcal{L}_{2}^{\prime} \mathcal{F}^{\mathcal{B}} \mid \mathcal{F}^{\mathcal{B} \dagger}\right\rangle}{\left\langle\mathcal{F}^{\mathcal{B}} \mid \mathcal{F}^{\mathcal{B} \dagger}\right\rangle}, \\
& b_{3}=\frac{\left\langle\mathcal{L}_{c}^{\prime \prime \prime} \mathcal{F}^{\mathcal{B}} \mathcal{F}^{\mathcal{B}} \overline{\mathcal{F}^{\mathcal{B}}} \mid \mathcal{F}^{\mathcal{B} \dagger}\right\rangle}{2\left\langle\mathcal{F}^{\mathcal{B}} \mid \mathcal{F}^{\mathcal{B} \dagger}\right\rangle}+\frac{\left\langle\mathcal{L}_{c}^{\prime \prime} \mathcal{X} \overline{\mathcal{F}^{\mathcal{B}}} \mid \mathcal{F}^{\mathcal{B} \dagger}\right\rangle}{\left\langle\mathcal{F}^{\mathcal{B}} \mid \mathcal{F}^{\mathcal{B} \dagger}\right\rangle}+\frac{\left\langle\mathcal{L}_{c}^{\prime \prime} \mathcal{F}^{\mathcal{B}} \mathcal{T} \mid \mathcal{F}^{\mathcal{B} \dagger}\right\rangle}{\left\langle\mathcal{F}^{\mathcal{B}} \mid \mathcal{F}^{\mathcal{B}}\right\rangle}, \\
& b_{4}=\frac{\left\langle\mathcal{L}_{c}^{\prime \prime} \mathcal{F}^{\mathcal{B}} \mathcal{Z} \mid \mathcal{F}^{\mathcal{B} \dagger}\right\rangle}{\left\langle\mathcal{F}^{\mathcal{B}} \mid \mathcal{F}^{\mathcal{B}^{\dagger}}\right\rangle}+\frac{\left\langle\mathcal{L}_{c}^{\prime \prime \prime} \mathcal{F}^{\mathcal{P}} \mathcal{F}^{\mathcal{P}} \mathcal{F}^{\mathcal{B}} \mid \mathcal{F}^{\mathcal{B} \dagger}\right\rangle}{2\left\langle\mathcal{F}^{\mathcal{B}} \mid \mathcal{F}^{\mathcal{B}^{\dagger}}\right\rangle} ; \\
& c_{1}=\frac{\left\langle\mathcal{F}^{\mathcal{G}} \mid \mathcal{L}_{1}^{\prime} \mathcal{F}^{\mathcal{P}}\right\rangle}{\left\langle\mathcal{F}^{\mathcal{G}} \mid \mathcal{F}^{\mathcal{P}}\right\rangle}, \quad c_{2}=\frac{\left\langle\mathcal{F}^{\mathcal{G} \dagger} \mid \mathcal{L}_{2}^{\prime} \mathcal{F}^{\mathcal{P}}\right\rangle}{\left\langle\mathcal{F}^{\mathcal{G}^{\dagger}} \mid \mathcal{F}^{\mathcal{P}}\right\rangle}, \\
& c_{3}=\frac{\left\langle\mathcal{F}^{\mathcal{G}} \mid \mathcal{L}_{c}^{\prime \prime} \mathcal{F}^{\mathcal{P}} \mathcal{Z}\right\rangle}{\left\langle\mathcal{F}^{\dagger} \mid \mathcal{F}^{\mathcal{P}}\right\rangle}+\frac{2 a_{1}\left\langle\frac{\partial \mathcal{F}^{\mathcal{G}}}{\partial \mathbf{r}} \mid \mathcal{Z}\right\rangle}{\left\langle\mathcal{F}^{\mathcal{G}} \mid \mathcal{F}^{\mathcal{P}}\right\rangle}+\frac{\left\langle\mathcal{F}^{\dagger} \dagger \mid \mathcal{L}_{c}^{\prime \prime \prime} \mathcal{F}^{\mathcal{P}} \mathcal{F}^{\mathcal{P}} \mathcal{F}^{\mathcal{P}}\right\rangle}{6\left\langle\mathcal{F}^{\mathcal{G}} \mid \mathcal{F}^{\mathcal{P}}\right\rangle}, \\
& c_{4}=\frac{\left\langle\mathcal{F}^{\mathcal{G} \dagger} \mid \mathcal{L}_{c}^{\prime \prime} \mathcal{F}^{\mathcal{P}} \mathcal{T}\right\rangle}{\left\langle\mathcal{F}^{\mathcal{G}} \mid \mathcal{F}^{\mathcal{P}}\right\rangle}+\frac{2 a_{1}\left\langle\frac{\partial \mathcal{F}^{\mathcal{G} \dagger}}{\partial \mathbf{r}} \mid \mathcal{T}\right\rangle}{\left\langle\mathcal{F}^{\mathcal{G} \dagger} \mid \mathcal{F}^{\mathcal{P}}\right\rangle}+\frac{\left\langle\mathcal{F}^{\mathcal{G}} \mid \mathcal{L}_{c}^{\prime \prime \prime} \mathcal{F}^{\mathcal{P}} \mathcal{F}^{\mathcal{P}} \overline{\mathcal{F}^{\mathcal{B}}}\right\rangle}{\left\langle\mathcal{F}^{\mathcal{G}} \mid \mathcal{F}^{\mathcal{P}}\right\rangle},
\end{aligned}
$$

and the functions $\mathcal{X}, \mathcal{Z}$ and $\mathcal{T}$ can be found from Eqn. (4.3) (4.8) and (4.9). Furthermore, the order parameter equations (4.10) can be modified to the more simple form by means of the linear transformation. The modified system reads:

$$
\begin{aligned}
\dot{\mathbf{R}} & =a_{1} \boldsymbol{\xi}, \\
\dot{\boldsymbol{\eta}} & =b \boldsymbol{\eta}+b_{3}|\boldsymbol{\eta}|^{2} \boldsymbol{\eta}+b_{4} \boldsymbol{\xi}^{2} \boldsymbol{\eta}, \\
\dot{\boldsymbol{\xi}} & =c \boldsymbol{\xi}+\widetilde{c_{3}} \boldsymbol{\xi}^{3}+\widetilde{c_{4}} \boldsymbol{\xi}|\boldsymbol{\eta}|^{2},
\end{aligned}
$$

where

$$
\begin{array}{rlrl}
b & =\varepsilon_{1} b_{1}+\varepsilon_{2} b_{2}, & c & =\varepsilon_{1} c_{1}+\varepsilon_{2} c_{2}, \\
\widetilde{c_{3}} & =c_{3}+\frac{3 a_{3} c}{a_{1}}, \quad \widetilde{c_{4}}=c_{4}+\frac{c+2 \operatorname{Re}(b)}{a_{1}} .
\end{array}
$$


A set of ordinary differential equations (4.11) is a system of order parameter equations, describing the dynamical behavior of a single dissipative soliton in the vicinity of the codimension-two bifurcation point. If the amplitude of the breathing mode $\boldsymbol{\eta}$ vanishes, the system (4.11) reads

$$
\begin{aligned}
\dot{\mathbf{R}} & =a_{1} \boldsymbol{\xi}, \\
\dot{\boldsymbol{\xi}} & =c \boldsymbol{\xi}+\widetilde{c_{3}} \boldsymbol{\xi}^{3},
\end{aligned}
$$

which can be recognized as a normal form of the drift-bifurcation [5], whereas $\boldsymbol{\xi}=0$ leads to a Hopf normal form for breathing dissipative solitons [7], i.e.,

$$
\dot{\boldsymbol{\eta}}=b \boldsymbol{\eta}+b_{3}|\boldsymbol{\eta}|^{2} \boldsymbol{\eta}
$$

Note that the first of the equations (4.11) indicates that the velocity of the soliton is completely defined by the temporal evolution of amplitude of the unstable propagator mode, whereas the last two equations show that evolution of $\boldsymbol{\eta}$ and $\boldsymbol{\xi}$ is $\mathbf{R}$-independent, which is a generic property of systems, featuring translational symmetry.

Using the exponential form of the complex amplitude $\boldsymbol{\eta}=\mathbf{A} e^{i \boldsymbol{\varphi}}$ one can rewrite (4.11) as

$$
\begin{aligned}
\dot{\mathbf{R}} & =a_{1} \boldsymbol{\xi}, \\
\dot{\boldsymbol{\xi}} & =c \boldsymbol{\xi}+\widetilde{c_{3}} \boldsymbol{\xi}^{3}+\widetilde{c_{4}} \boldsymbol{\xi} \mathbf{A}^{2}, \\
\dot{\mathbf{A}} & =\operatorname{Re}(b) \mathbf{A}+\operatorname{Re}\left(b_{3}\right) \mathbf{A}^{3}+\operatorname{Re}\left(b_{4}\right) \boldsymbol{\xi}^{2} \mathbf{A}, \\
\dot{\boldsymbol{\varphi}} & =\operatorname{Im}(b)+\operatorname{Im}\left(b_{3}\right) \mathbf{A}^{2}+\operatorname{Im}\left(b_{4}\right) \boldsymbol{\xi}^{2}
\end{aligned}
$$

We are interested in the second and the third equations of (4.12). This system admits a trivial solution $\mathbf{A}_{0}=0, \boldsymbol{\xi}_{0}=0$, corresponding to the case of the stationary solution $\mathbf{q}_{0}$. The trivial solution is stable if

$$
\varepsilon_{1} \operatorname{Re}\left(b_{1}\right)+\varepsilon_{2} \operatorname{Re}\left(b_{2}\right)<0, \quad \varepsilon_{1} c_{1}+\varepsilon_{2} c_{2}<0 .
$$

Apart from the trivial solution $\left(\mathbf{A}_{0}, \boldsymbol{\xi}_{0}\right)$, one can also find three nontrivial solutions of the form

$$
\begin{aligned}
& \mathbf{A}_{1}=\sqrt{-\frac{\operatorname{Re}(b)}{\operatorname{Re}\left(b_{3}\right)}}, \quad \boldsymbol{\xi}_{1}=0, \\
& \mathbf{A}_{2}=0, \quad \boldsymbol{\xi}_{2}=\sqrt{-\frac{c}{\widetilde{c}_{3}}}, \\
& \mathbf{A}_{3}=\sqrt{\frac{c \operatorname{Re}\left(b_{4}\right)-\widetilde{c}_{3} \operatorname{Re}(b)}{\widetilde{c}_{3} \operatorname{Re}\left(b_{3}\right)-\widetilde{c}_{4} \operatorname{Re}\left(b_{4}\right)}}, \quad \boldsymbol{\xi}_{3}=\sqrt{\frac{\widetilde{c}_{4} \operatorname{Re}(b)-c \operatorname{Re}\left(b_{4}\right)}{\widetilde{c}_{3} \operatorname{Re}\left(b_{3}\right)-\widetilde{c}_{4} \operatorname{Re}\left(b_{4}\right)}},
\end{aligned}
$$

where $\left(\mathbf{A}_{1}, \boldsymbol{\xi}_{1}\right)$ corresponds to a breathing soliton solution (see Fig. 4 (a)), $\left(\mathbf{A}_{2}, \boldsymbol{\xi}_{2}\right)$ represents solution, moving with the constant velocity (see Fig. 4 (b)) and $\left(\mathbf{A}_{3}, \boldsymbol{\xi}_{3}\right)$ stands for dissipative soliton, moving with the constant velocity and breathing with the constant amplitude (see Fig. 4 (c)). The collapse of the soliton, presented in Fig 4 (d) corresponds to the first fixed point in the absence of nonlinear stabilization.

\subsection{Order parameter equations for a three-component reaction-diffusion system}

Now let us apply general results, obtained in the previous subsection, to the three-component reactiondiffusion system (1.1). As we mentioned above, the linearization operator $\mathcal{L}^{\prime}\left(\mathbf{q}_{0}, \nabla, \boldsymbol{\sigma}\right), \boldsymbol{\sigma}=(\tau, \theta)$ is not self-adjoint. Nevertheless, for the systems like (1.1) it can be represented as a product

$$
\mathcal{L}^{\prime}\left(\mathbf{q}_{0}, \nabla, \boldsymbol{\sigma}\right)=M L\left(\mathbf{q}_{0}, \nabla\right)
$$


of an invertible matrix

$$
M:=M(\boldsymbol{\sigma})=\left(\begin{array}{ccc}
1 & 0 & 0 \\
0 & -1 / \kappa_{3} \tau & 0 \\
0 & 0 & -1 / \kappa_{4} \theta
\end{array}\right)
$$

and a self-adjoint operator

$$
L\left(\mathbf{q}_{0}, \nabla\right)=\left(\begin{array}{ccc}
D_{u} \Delta+\lambda-3 u_{0}^{2} & -\kappa_{3} & -\kappa_{4} \\
-\kappa_{3} & -\kappa_{3} D_{v} \Delta+\kappa_{3} & 0 \\
-\kappa_{4} & 0 & -\kappa_{4} D_{w} \Delta+\kappa_{4}
\end{array}\right) .
$$

In order to estimate all coefficients of the normal form (4.11), the critical eigenfunctions of the operators $\mathcal{L}^{\prime}$ and $\mathcal{L}^{\prime \dagger}$ at the bifurcation point $\boldsymbol{\sigma}_{c}=\left(\tau_{c}, \theta_{c}\right)$ should be calculated. In general, the analytical calculations of the spectrum of the adjoint operator $\mathcal{L}^{\prime \dagger}$ are difficult, but in the case of reaction-diffusion system we are interested in, it is possible due to special form (4.16) of the operator $\mathcal{L}^{\prime}\left(\mathbf{q}_{0}, \nabla, \boldsymbol{\sigma}\right)$. For instance, the eigenfunctions $\mathcal{F}^{\mathcal{B} \dagger}$ and $\mathcal{F}^{\mathcal{G} \dagger}$ can be found as

$$
\mathcal{F}^{\mathcal{B} \dagger}=M_{c}^{-1} \overline{\mathcal{F}^{\mathcal{B}}}, \quad \mathcal{F}^{\mathcal{G} \dagger}=M_{c}^{-1} \mathcal{F}^{\mathcal{G}},
$$

where $M_{c}=M\left(\sigma_{c}\right)$. Moreover, one can also find the analytical relation for the generalized eigenfunction $\mathcal{F}^{\mathcal{P} \dagger}$ in terms of the modes of $\mathcal{L}_{c}^{\prime}$, namely

$$
\mathcal{F}^{\mathcal{P} \dagger}=M_{c}^{-1} \mathcal{F}^{\mathcal{P}}+\vartheta M_{c}^{-1} \mathcal{F}^{\mathcal{G}},
$$

where the constant $\vartheta$ can be found by applying the solvability condition $\left\langle\mathcal{F}^{\mathcal{P}} \mid \mathcal{F}^{\mathcal{P}}\right\rangle=0$ at the bifurcation point, i.e.,

$$
\vartheta=-\frac{\left\langle M_{c}^{-1} \mathcal{F}^{\mathcal{P}} \mid \mathcal{F}^{\mathcal{P}}\right\rangle}{\left\langle\mathcal{F}^{\mathcal{P} \dagger} \mid \mathcal{F}^{\mathcal{G}}\right\rangle} .
$$

That is, using relations (4.17) and (4.18) one can calculate all complex coefficients of the system (4.11) in terms of the critical eigenfunctions of the operator $\mathcal{L}_{c}^{\prime}$ only. After that the system (4.11) can be solved numerically and compared with results, obtained from direct numerical simulations of the system (1.1). An example is presented on Fig. 5, where the time evolution of the position of the soliton $\mathbf{R}(t)$ in shown

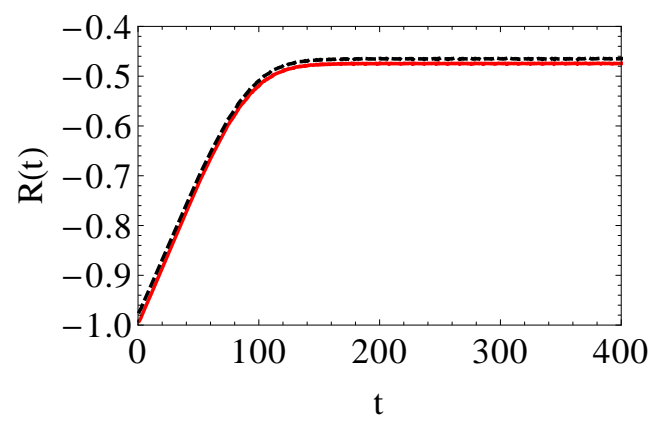

Figure 5. Temporal evolution of the position $\mathbf{R}(t)$ of the soliton calculated from the system (4.11) (dashed black line) and as result of direct numerical simulation of the system (1.1).

(red curve) and compared with the solution of Eq. (4.11) (black dashed line). Both solution correspond to the case, where in the short run the velocity of the soliton becomes zero and breathing amplitude reaches the constant value. 


\section{Conclusion}

In this paper we have presented analytical and numerical investigations of moving and breathing dissipative solitons in a three-component reaction-diffusion system. We have shown that depending on control parameters, different combinations of movement and breathing can be observed in the vicinity of the codimension-two bifurcation point. In particular, a solution in form of a soliton, moving with a constant velocity and breathing with a constant amplitude is observed. This situation is analyzed by performing multiple scale perturbation expansion in the vicinity of the codimension-two bifurcation point, and a set of order parameter equations, explicitly describing the dynamics of the single dissipative soliton in the vicinity of the bifurcation point is derived. Information about the behavior of the system in now contained in the complex coefficients of these equations. In addition, presented analytical results are obtained in general form and can be extended to other systems.

\section{References}

[1] R. Woesler, P. Schütz, M. Bode, M. Or-Guil, H.-G. Purwins. Oscillations of fronts and front pairs in two- and threecomponent reaction-diffusion systems. Physica D, 91 (1996), 376-405, 1996.

[2] C. P. Schenk, M. Or-Guil, M. Bode, H.-G. Purwins. Interacting pulses in three-component reaction diffusion systems on two-dimensional domains. Physical Review Letters, 78 (1997), 3781-3784.

[3] R. Kapral, K. Showalter, editors. Chemical Waves and Patterns, vol. 10 of Understanding Chemical Reactivity, Kluwer Academic Publishers, Dordrecht, 1995.

[4] M. A. Dahlem, R. Graf, A. J. Strong, J. P. Dreier, Y. A. Dahlem, M. Sieber, W. Hanke, K. Podoll, E. Schöll. Two-dimensional wave patterns of spreading depolarization: Retracting, re-entrant, and stationary waves. Physica D: Nonlinear Phenomena, 239 (2010), 889 - 903.

[5] H.-G. Purwins, H.U. Bï ¿ $\frac{1}{2}$ deker, Sh. Amiranashvili. Dissipative solitons. Advances in Physics, 59 (2010), $485-701$.

[6] H. Engel, F.-J. Niedernostheide, H.-G. Purwins, E. Schöll. Self-Organization in Activator-Inhibitor-Systems: Semiconductors, Gas-Discharge and Chemical Active Media. Wissenschaft und Technik Verlag, Berlin, 1996.

[7] S. V. Gurevich, Sh. Amiranashvili, H.-G. Purwins. Breathing dissipative solitons in three-component reaction-diffusion system. Physical Review E, 74 (2006) 066201.

[8] M. Or-Guil, M. Bode, C. P. Schenk, H.-G. Purwins. Spot Bifurcations in Three-Component Reaction-Diffusion Systems: The Onset of Propagation. Physical Review E, 57 (1998), 6432-6437.

[9] M. Bode, A. W. Liehr, C. P. Schenk, H.-G. Purwins. Interaction of Dissipative Solitons: Particle-like Behaviour of Localized Structures in a Three-Component Reaction-Diffusion System. Physica D, 161 (2002), 45-66.

[10] M. Bode. Front-bifurcations in reaction-diffusion systems with inhomogeneous parameter distribution. Physica D, 106 (1997), 270-286.

[11] P. Schütz, M. Bode, V. V. Gafiichuk. Transition from stationary to travelling localized patterns in a two-dimensional reaction-diffusion systems. Physical Review E, 52 (1995), 4465-4473

[12] A. Ankiewicz N. Akhmediev, editors. Dissipative Solitons. Volume 661 of Lecture Notes in Physics, Springer, Berlin, 2005.

[13] A. Ankiewicz N. Akhmediev, editors. Dissipative Solitons: From Optics to Biology and Medicine. Volume 751 of Lecture Notes in Physics, Springer, Berlin, 2008.

[14] A. S. Mikhailov, K. Showalter. Control of waves, patterns and turbulence in chemical systems. Physics Reports, 425 (2006), $79-194$.

[15] C.I. Christov, M.G. Velarde. Dissipative solitons. Physica D: Nonlinear Phenomena, 86 (1995), 323 - 347.

[16] R. Richter, A. Lange. Surface Instabilities of Ferrofluids. in S. Odenbach (editor), Colloidal Magnetic Fluids, vol. 763 of of Lecture Notes in Physics, Springer Berlin, 2009.

[17] O. Lioubashevski, Y. Hamiel, A. Agnon, Z. Reches, J. Fineberg. Oscillons and propagating solitary waves in a vertically vibrated colloidal suspension. Physical Review Letters, 83 (1999), 3190.

[18] P. B. Umbanhowar, F. Melo, H. L. Swinney Localized excitations in a vertically vibrated granular layer Nature 382 (1996), 793-796

[19] H. Haken. Synergetics. Introduction and Advanced Topics, Springer, Berlin, 1983. 